\title{
Index of CyberResearch* (including computer science, digital practice, mathematical, and technological terms)
}

$$
\begin{array}{ll}
2 \mathrm{D} & 284,304-305 \\
\text { 3D } & 37,284 \mathrm{n} 6,305
\end{array}
$$

A-Temporal Diffusion Model (ATDM) 66n27, 71

AAS (Artificial Adaptive System) 61, 63, 66

Abstract vector $\quad 297,297 n_{54}, 298,304$

See also features' vector; Optical Character Recognition

See also in General Index sign

Adab network graph 210n59, 211

Adjacency matrix $\quad 273-275,306 \mathrm{n} 87$

See also edge; node

AI (Artificial Intelligence) 62, 63n12, 307, 344,347

Algorithm 6on2, 61n5, 63n12, 64n16-17, 65n2o, 66-67, 70, 91-92, 94-95, 98, 106, 118, 120-122, 133-134, 197, 206, 209, 213-214, 217-220, 26o, 261n10, 275, 277, 296, 304, 307-308, 338, 344

See also Artificial Neural Network

American Standard Code for Information Interchange (ASCII) characters $\quad 285$, 287-288

Analyse logiciste $\quad 87$

Analytical taxonomies 154,161-162, 166, 181-182, 185-186

ANN (Artificial Neural Network) 62ng, 64, 66, 94-95, 229, 246, 261

Application Programming Interface (API) 290, 318

See also Online Cultural and Historical Research Environment

\author{
Arbitrary unit 39 \\ See also in General Index spatial unit
}

Arc 74, 308n93, 347

Architecture (in CyberResearch) 73n36, 248, 291n33, 297n54, 307, 321, 338

Artificial Adaptive Systems (AAS) $\quad 61,63,66$

See also algorithm; Artificial Sciences; Natural Computing

Artificial Intelligence (AI) 62, 63n12, 307, 344,347

See also Artificial Neural Network

Artificial intelligence model $\quad 63 n 12$

See also Mesopotamian Urban Revolution Landscape

Artificial Neural Network (ANN) 62ng, 64, 66, 94-95, 229, 246, 261

See also algorithm; Artificial Intelligence; neural networks; Self-Organizing Map; Word2vec

Artificial Sciences (AS) $\quad 6 \mathrm{in}_{5}$

See also Artificial Adaptive Systems As (Artificial Sciences) $6 \mathrm{in}_{5}$ ASCII characters (American Standard Code for Information Interchange characters) $\quad 285,287-288$

ASCII Transliteration Format (ATF) 200, 285n11

See also American Standard Code for Information Interchange characters; Cuneiform Digital Library Initiative; Open Richly Annotated Cuneiform Corpus

See also in General Index tablet

* The words following the first word of a term are capitalized when the expression has an acronym but not capitalized when the expression has no acronym. The related entries after "see also" follow the same rule for acronyms but are not capitalized when the expression does not have an acronym. There are some exceptions, such as Python, R, and Word2vec, that are applications or tools, and thus always capitalized. The index entries followed by "see also" refer to related term(s)/expression(s) in glossaries. 
ATDM (A-Temporal Diffusion Model) 66n27, 71

ATF (ASCII Transliteration Format) 200, 285n11

Atomization $314 \mathrm{n} 4,319,323$

See also in General Index logosyllabic; sign

Attribute (substantive) 34, 43-44, 46, 47n84, 50-51, 118-121, 123-124, 127, 134, 136-137, 164-166, 171ng2, 172n95, 182, 202n35, 220, 289, 295, 302-303, 309

See also edge; node; tag; Text Encoding Initiative

Attribute-analysis approach 118-119

Augmenting a dataset $\quad 307 n 91$

Auto-CM (Auto-Contractive Map) 66n26, 68-74

Auto-Contractive Map (Auto-CM) 66n26, 68-74

See also Artificial Neural Network

Automatic conversion 290

BabelNet 170

Bag-of-words model 270-271

See also Continuous Bag-of-Words model

Bavant-XML 286-287, 291, 294n43, 295

Big data $\quad 156$

Bigram 239-241, 246

Binary correspondence analysis 99

Bridge 202, 212, 217

See also edge betweenness; graph partitioning; node

See also in General Index Adab corpus

$\mathrm{C}++$ language $\quad 120,257 \mathrm{n} 2$

C(anonical)-ATF (C-ATF) 199n18, 199n23, 2oon24, 205n47, 285-286, 291, 292n36, 294n43, 295

See also American Standard Code for Information Interchange characters; Cuneiform Digital Library Initiative C-ATF (C(anonical)-ATF) 199n18, 199n23, 2oon24, 205n47, 285-286, 291, 292n36, 294n 43,295

CAL (Comprehensive Aramaic Lexicon) 288, $292 n 34$

CAL Code ～288-289, 291, 294n43, 295

See also American Standard Code for Information Interchange characters

See also in General Index Aramaic cBow model (Continuous Bag-of-Words model) 247-248
CDLI (Cuneiform Digital Library Initiative) 88, 198-200, 202n37, 203, 218n79, 285-286, 346n24, 352, 355, 36o

CIDOc Conceptual Reference Model (CIDOC CRM) $\quad 45,48,300,336,353-357,361$

See also Conceptual Reference Model; International Organization for Standardization; ontology

CIDOC CRM (CIDOC Conceptual Reference Model) 45, 48, 300, 336, 353-357, 361

Class $\quad 42,45 \mathrm{n} 71,64 \mathrm{n} 17,65 \mathrm{n} 20,94,119$, 122-124, 127, 291n33, 300, 308, 347-348, $354-361$

Class identifiers 301 Classical Language Toolkit (CLTK) 283n2 Classification of ontologies 348

Clique 214-216

See also maximal clique; network graph analysis; node

See also in General Index tablet

Close reading 156 n3o, 233, 259

CLTK (Classical Language Toolkit) 283n2

Cluster 119-120, 123-124, 133-134, 212, 220n87, 246, 258, 275-276, 278, 338, 345

Cluster analysis 118,274

See also algorithm; multivariate analysis

Clustering method 214n68, 220, 274

See also cophenetic correlation coefficient; network analysis; node; social network analysis; Unweighted Pair-Group Average

CNNs (Convolutional Neural Networks) 307ng1

Comma Separated Value (CSv) 137, 206, 274, 344

Comprehensive Aramaic Lexicon (CAL) 288, 292n34

Computer-aided textual analysis $\quad$ 258-259

Computer Science $\quad 60,197 n 16,299,343$

Computer semiotics 60

Computerized data modeling 55

See also data model

Conceptual Reference Model (CRM) 45, 300

See also cIDoc Conceptual Reference

Model; Data Management System;

Linked Open Data; ontology

Continuous Bag-of-Words (своw) model 247-248

See also bag-of-words model; Word2vec 
Continuous Skip-gram model 247

See also Word2vec

Convolutional layer 307ng1

Convolutional Neural Networks (CNNs) $307 n 91$

Cophenetic correlation coefficient 274 See also clustering method

Correspondence analysis $\quad$ 94-95, 99, 105-106 See also hierarchical classification; quantitative analysis/method

Cosine similarity $\quad 272-274$

See also Document Term Matrix

CountVectorizer 271

CRM (Conceptual Reference Model) 45, 300

Cross-platform 304

CSv (Comma Separated Value) 137, 206, 274, 344

Cultural heritage data $45,353,355,361$

Cuneiform Digital Library Initiative (CDLI) 88, 198-200, 202n37, 203, 218n79, 285-286, 346n24, 352, 355, 36o

See also American Standard Code for Information Interchange characters; metadata

See also in General Index cuneiform; transliteration

Cuneiform Digital Palaeography Project

Cytoscape 209, 210n59, 211n61, 212-213, $218 n 80$

See also Gephi; graphing programs; weighted

DANA (Digital Archaeology and National Archive) $\quad 28$

Data-management system $\quad 36 n_{52}, 38 n_{55}, 45$

Data cloud 49, 55

See also graph; Linked Open Data; triple store

Data mining 91n21, 162, 181, 26on8, 344

See also Système Portable pour l'Analyse des Données; text mining

Data model $48-49,55,154 \mathrm{n} 15,315,319-320$, 323, 330, 333-334, 339

See also computerized data modeling

Data processing 181,257n2, 268

Data serialization 299, 305

See also External Data Representation; JavaScript Object Notation
Data structure $\quad 47 \mathrm{n} 83,48 \mathrm{ng}$ 51ng8, 196-197, 202, 297n55, 306, 345, 347

Database $\quad 27-29,32,46 \mathrm{n} 82,47,49,51,67 \mathrm{n} 28$, 114, 119, 123, 136-137, 170, 171ng2, 174, 181, 199-200, 204-206, 285, 290, 314n3-4, $315,317-326,329-330,333-334,345,360$

Database Management System (DBMS) $47 \mathrm{n} 84$

See also Conceptual Reference Model; identifier; meta-system

Database of Neo-Sumerian Texts 351

Dataset 25, 27, 46-48, 49n95, 50, 55, 65n2o, 66n27, 67n28, 71, 74, 90, 91n21, 92, 94n26, 95-100, 106, 117-118, 121-122, 124, 128, 133-135, 137, 156n3o, 162, 198, 209, 212, 219-220, 229n17, 259n4, 26o-261, 267-268, 271-276, 307, 319n23, 336, 341, 343-344, 347-349

DBMs (Database Management System) $47 \mathrm{n} 84$

Deep learning $\quad 66,225 n 6,296,298 n 57$

Diachronic Corpus of Sumerian Literature 351

Dictionary $\quad 203,297$ n55, 321, 324

See also map

Digital Archaeology and National Archive (DANA) 28

Digital Library Development Center (University of Chicago) $\quad 318$

Digital theodolite $27 \mathrm{n} 15$

Distant reading $\quad 259-260$

Distributional semantic models 228 See also language technology; Pointwise Mutual Information; Word2vec

Distributional semantics $229 n 17$

Document-oriented database $\quad 5^{1}$

See also tag

Document Term Matrix (DTM) 271-272, 274, $284 n 6$

See also cosine similarity; vector space

DTM (Document Term Matrix) 271-272, 274, $284 n 6$

Dublin Core $\quad 353,354 n_{56}$

Edge $38,46 \mathrm{n} 80,67 \mathrm{n} 28,73,104,201-202,206$, 207n $52,208-212,216-217,220,253,260$, 275, 305-306

See also graph; label; node 
Edge betweenness 212, 217

See also bridge; edge; network analysis

Ego-network 209, 210n59, 217, 219

See also Gephi; network graph; node

Electronic Text Corpus of Sumerian Literature (ETCSL) 204, 336, 342, 350-356, 358-6o, 36on74-75, 361

See also American Standard Code for Information Interchange characters; Unicode

See also in General Index Sumerian; transliteration

Element $36,62 n 9,67 \mathrm{n} 28,100,104,164-165$, 167, 168n78, 169, 171ng2, 174, 182, 184, 204, 239, 259, 293, 294n43, 301-302, 303n $76,306 \mathrm{n} 88$

See also attribute; markup; tag; Text Encoding Initiative

Elementary semantic unit $\quad 285 \mathrm{n} 8,291 \mathrm{n} 32$ Encode/encoding $\quad 60-63,64 \mathrm{n} 17,72,87-88$, 91-93, 96-97, 100, 152, 154, 164-165, 167 , 199n18, 199n23, 200, 204n42, 22on86, 267n27, 284-286, 289-294, 297-304, 307-309, 326n33, 328, 339

Encoding process $\quad 152,285 \mathrm{n} 10,289 \mathrm{n} 27$ Encoding scheme $\quad 200,205 n_{48}, 284 n_{5}$, 286n14, 290-293, 295-296, 297n 52 , 298-301, 304-305, 306ngo, 307-310

See also interoperability; minimal semantic unit

Entity $30,37 \mathrm{n}_{52}, 3^{8}-39,42-44,46-47$, 49-5o, 55, 71, 204, 206, 209n $54,215^{-216}$, $339,345,347-349,354,35^{8-359}$

Entity-relationship model 355

EpiDoc $285,289-291,294 n 43,295,298-304$, 308-310

See also Extensible Markup Language; Text Encoding Initiative; Unicode Transformation Format (UTF)-8

Epigraphic interoperability $284 \mathrm{n}_{5}, 296$, 299n63, 305, 308n95, 309

See also interoperability

ePSD (Pennsylvania Sumerian Dictionary) $35^{2}$

ETCSL (Electronic Text Corpus of Sumerian Literature) 204, 336, 342, 350-356, $35^{8-6 o, 36 o n} 74-75,361$

Euclidean metric $\quad 121$
Event $\quad 36-37,39-40,42,44,50,66,74$

See also syntactic elements

Extensible Markup Language (XML) 51n98, 163-164, 203-204, 289n27, 299, 302n72, 303-305, 319, 334, 339-340, 347

See also data serialization; EpiDoc; interoperability; machine-actionable data/machine-readable data; markup; RDF-XML

Extensible Markup Language (XML) annotation 204

See also Electronic Text Corpus of Sumerian Literature; markup; Text Encoding Initiative; web

Extension 45, 122, 219n82, 295-296, 353, 36o External Data Representation (XDR) 299

See also data serialization; JavaScript Object Notation

Extract/Extracting 91n21, 163,166-167, 181, 194-197, 204-206, 219, 276, 285ng, 292, 293n41, 296n5o, 301, 304n83, 318n18, 321n27, 322n29

Extraction/extractor 91n21, 161-163, 197, 293n4o, 304n83, 308

Factor(s) 95n29, 100, 115n21, 127, 210n6o, 217, 228, 263

Factor analysis $\quad 115,119$

Factors of variance/factors of variation 308ng3

See also feature extraction algorithm

Feature(s) 36, 37n54, 54, 65n2o-21, 67n28, 91n21, 94n26, 94n28, 209, 293n40, 295n46, 296n49, 297, 308, 314n2, 318n17, $35^{\circ}, 353$

Feature extraction algorithm $\quad 304 n 83,308$

See also algorithm; factors of variance

Features' vector $\quad 45$ n71, 297-300, 304, 308

See also in General Index sign

FileMaker 114, 124

FOAF ontology 358

Format $46-47,98,106,137,196 \mathrm{n} 7,200,204$, 205n47, 206n49, 209, 267, 274n36, 295n47, 299, 3oon64, 30on66, 301, 303, $305,314,319 n 21,336,344,348$

FrameNet 170

FRBR (Functional Requirements for Bibliographic Records) 355 
FRBRoo $336,353-357,361$

See also cIDoc Conceptual Reference

Model; Functional Requirements for

Bibliographic Records; ontology

Frequency analysis 134

Frequency counts $27 \mathrm{in} 34$

Function $\quad 60-62,67 \mathrm{n} 28,87 \mathrm{n} 4,97 \mathrm{n} 35$,

205n 47, 213-214, 268, 270-272, 303n 77 ,

305-306, 314, 351

Functional Requirements for Bibliographic Records (FRBR) 355

See also ciDoc Conceptual Reference

Model; FRBRoo

Gaussian distribution 121, 133

Gephi 209, 210n59, 211n61, 213n65, 218n8o, 253, 274

See also Cytoscape; edge; ego-network; graph data; graphing programs; network graph; node

Graph 46n8o, 51, 67n28, 99, 211, 206, 213n65

See also edge; Gephi; graph data; node

Graph-algorithms 66

Graph analysis $\quad 66-67,201,214$

Graph data $\quad 46-47,49,197,202,209,210 n_{59}$, 219-220

See also Gephi; graph

Graph database $\quad 46-47,49-51,55$

Graph model 46

See also edge; node; triple

Graph partitioning 212

See also bridge; graph; quantitative analysis/method

Graph theory $\quad 46 \mathrm{n} 80,66 \mathrm{n} 24,73,305 \mathrm{n} 84,307$

Graph visualization software 208

See also Cytoscape; Gephi

Graphic 94, 162, 182n127, 304

Graphical User Interface (GUI) 97n35, 303-304

See also data mining; Système Portable pour l'Analyse des Données

Graphing programs 274

See also Cytoscape; Gephi

GUI (Graphical User Interface) 97n35, 303-304

Hierarchical classification $\quad$ 94-95

See also correspondence analysis
Hierarchical clustering algorithm 120-121, 133

Hierarchical softmax $\quad 247-248$

See also negative sampling; Word2vec

HTML (HyperText Markup Language) 204n43, 338-339, 342, 352

HTTP (HyperText Transfer Protocol) 338-340

HTTP URIs (HyperText Transfer Protocol Universal Resource Identifiers) 344, $346-348$

Hub 210

See also network graph; node

Human-oriented 292

Human readable $287,290 \mathrm{n} 27,303,346 \mathrm{n} 25$, 347

Hyperlinks 338

See also HyperText Transfer Protocol; web HyperText Markup Language (HTML) 204n 43, 338-339, 342, $35^{2}$

See also HyperText Transfer Protocol

HyperText Transfer Protocol (HTTP) 338-340

See also HyperText Markup Language; HyperText Transfer Protocol Universal Resource Identifiers; web

HyperText Transfer Protocol Universal Resource Identifiers (HTTP URIs) 344, $346-348$

See also HyperText Markup Language; HyperText Transfer Protocol; Semantic Web; Universal Resource Identifiers; web

ID (Identifier) 36-37, 43-44, 47n84, 50, 55, 123, 196n $7,202 \mathrm{n} 35,204,207-208,268$, 301, 308, 338-339

See also Database Management System Identifier (ID) $36-37,43-44,47 \mathrm{n} 84,50,55$, 123, 196n7, 202n 35, 204, 207-208, 268, 301, 308, 338-339

IFLA (International Federation of Library Associations and Institutions) 355

Inheritance 291333

Input $64 \mathrm{n} 16,65 \mathrm{n} 20-21,66 \mathrm{n} 25,72,73 \mathrm{n} 36$, 252, 261n10, 268, 271, 274, 285n9, 293, 295-296, 298, 304n83

Instance $\quad 47 \mathrm{n} 84,270,345,347,354,35^{6-360}$ 
Interchangeable 344

International Federation of Library

Associations and Institutions (IFLA) 355

International Organization for

Standardization (ISO) $\quad 45,353,355$,

357

Interoperability/epigraphic interoperability 29, 164, 284-285, 291-292, 296, 299n63, 305, 308n95, 309

See also encoding scheme

Iso (International Organization for

Standardization) $\quad 45,353,355,357$

Item 42n69, 120, 123, 184, 229n17, 271, 275,

$314 n 4,318-325,333,352,354,35^{6}$

Iterative process $120,257,270$

Java $318 \mathrm{n} 17$

Java application client/Java client $\quad 318,334$

See also Java Web Start; Online Cultural

and Historical Research Environment

Java Web Start 318

See also Online Cultural and Historical

Research Environment

JavaScript $35^{2}$

JavaScript Object Notation (JSON) 299, 305, 340

See also data serialization; External Data Representation

JSON (JavaScript Object Notation) 299, 305, 340

JSON-LD 340

k-plex 216, 220

See also edge; maximal clique; node

Key 297n55, 319-320

Knowledge Representation (KR) 315, 344, 347

KR (Knowledge Representation) 315, 344, 347

Label $36 n_{52}, 202,267,274$

See also attribute; edge; identifier; node Language technology $\quad 225^{-228,231}$

See also distributional semantic models

Language-technology-related research $\quad 227$

Layer $\quad 73,195,204,246-247,307 n 91$
LD (Linked Data) 47, 55, 201n39, 300, 308, 310, 336, 338, 340, 343-345, 346n25, 347-349, 352, 355n6o, 36o

Learning process $\quad 296 n_{51}, 298 n_{56}$

Lemmatization 197, 203

Library 218n79, 268, 270, 274, 283n2

See also Cuneiform Digital Library Initiative

Linked $\quad 46-48,74,216,319,321,328,330,354$

See also Online Cultural and Historical Research Environment; web

Linked Data (LD) 47, 55, 201n39, 300, 308, $310,336,338$, 340, 343-345, 346n25, 347-349, 352, 355n6o, 360

See also edge; HyperText Transfer Protocol Universal Resource Identifiers; network graph; ontology; Simple Knowledge Organization System

Linked Data cloud 46

Linked Open Data (LOD) 45, 49n95, 344

See also Linked Data; Open Data; Resource Description Framework

Linked Open Data cloud 45 LOD (Linked Open Data) 45, 49n95, 344 $\log (\operatorname{logarithm}) \quad 239$

Logarithm 239

See also Pointwise Mutual Information

Machine-actionable/machine-readable 46, 164, 200, 204n41, 217, 220, 326n, 336, 339-340

Machine-actionable data/machine-readable data 20on27

See also scraper

Machine learning $\quad 72-73,194,217,260 n 8$, 296n5o, 298n57

Machine-readability 344

Machine-understanding 344

Manuel de Codage (MdC) 287-288, 291, 292n36, 293n4o, 294n43, 295-296, 298, 309

See also American Standard Code for Information Interchange characters

Map/mapping/to map (in the context of a dictionary-data structure) $\quad 246,289$, 296, 297n55, 298, 300, 354, 357-358

See also dictionary 
Map/mapping (in other contexts) 30, 45, 47-49, 65n20-21, 71-72, 94n26, 94n28, 120, 309, 354-360

MapReduce model $\quad 220 n 87$

Markup/markup tag/markup tagging 163 , 165-166, 186, 204, 289-290, 299, 302n73

See also element; Extensible Markup Language annotation; HyperText Markup Language; tag

Markup language $163,203,204 \mathrm{n} 42-43,259$, 289

Markup scheme 165

Matrix/matrices $\quad 62-64,66,72-74,271-275$, 296, 299, 306

Maximal cliques $\quad 214-216$

See also clique; $k$-plex

Maximally Regular Graph (MRG) $\quad 67,69,74$

See also Minimum Spanning Tree

See also in General Index Mesopotamian

Urban Revolution Landscape

Maximally Regular Graph (MRG) algorithm 67

See also graph; Maximally Regular Graph; Minimum Spanning Tree

Meta-model 35

See also model

Meta-system 37, 38n55

See also Data Management System

Metadata $36,38,199,220,225,228,253,323$, $339,341,343,345 \mathrm{n} 23,353,35^{6}$

See also Cuneiform Digital Library

Initiative; Open Richly Annotated Cuneiform Corpus

Method 120

Metric scaling 120

Minimal semantic unit $\quad 286,292$

See also encoding scheme

Minimum Spanning Tree (MST) 67, 69-70, 73-75

See also edge; graph; Maximally Regular Graph

Minimum Spanning Tree (MST) data mining $67 \mathrm{n} 28$

See also data mining; Minimum Spanning Tree; spatial analysis

Mixed method 163

See also qualitative method; quantitative analysis/method
Model (substantive) $\quad 30,32,35,45^{-46}$, 48ng1,49, 51, 61, 154n15, 196, 204, 220n87, 229, 247, 270-271, 300n64, 310, 315 , 319-320, 330, 333-334, 339, 348, 355

See also meta-model

MRG algorithm (Maximally Regular Graph algorithm) 67

MST (Minimum Spanning Tree) 67, 69-70, 73-75

MST data mining (Minimum Spanning Tree data mining) $67 \mathrm{n} 28$

Multivariate-attribute-analysis approach $\quad 118$

Multivariate analysis $\quad 65 \mathrm{n} 20,118$

MySQL 196, 206-207, 218n8o

See also querying; relational database; Structured Query Language

Namespace $\quad 295 \mathrm{n} 45,302,308$

See also EpiDoc; tag

Natural Computing (NC) 6o, 6in $5,61 n_{7}$, $63 n 12$

See also Artificial Adaptive System; Natural Computing algorithms

Natural Computing (NC) algorithms 6on2

See also algorithm; Natural Computing; neural networks

Natural Language Processing (NLP) 194, 238, $246,322,344$

Natural Language Toolkit (NLTK) $\quad 238$

NC (Natural Computing) 6o, 6in $5,6 i n_{7}$, $63 n 12$

NC algorithms (Natural Computing algorithms) 6on2

Negative sampling $\quad 247-248$

See also hierarchical softmax; Word2vec

Network analysis 61n4, 194-197, 201-202, 212n62, 214, 216-217, 219-221, 227

See also quantitative analysis/method

Network analysis algorithms 197

See also algorithm; network analysis

Network graph 196-197, 201, 202, 206, 208-211, 213-214, 216-217, 220

See also edge; graph; node; triple

Network graph analysis 201, 214

See also graph; network graph

See also in General Index administrative texts

Network graph triples 206

See also edge; network graph; node; triple 
Network graph visualization 208, 210

See also graph; network graph

Network theory 195

See also network graph; social network analysis

Neural networks $\quad 60 \mathrm{n} 2,62 \mathrm{ng}, 229 n 19,307 \mathrm{ng} 1$

See also Artificial Neural Network; Natural Computing algorithms

NLP (Natural Language Processing) 194, 238, 246, 322, 344

NLTK (Natural Language Toolkit) $\quad 238$

Node 46n8o, 61, 65n2o, 66, 67n28, 73, 168, 180, 196, 201-202, 206, 209-214, 216, 220, 229n19, 253, 260, 305-306, 347

See also edge; edge betweenness; ego-network; network analysis; Self-Organizing Map; weighted See also in General Index Mesopotamian Urban Revolution Landscape

Normalization 199, 293, 295-296, 304, 307, 309

Normalization process $\quad$ 293, 295-296, 309

Normalized Pointwise Mutual Information (NPMI) 240-246

See also logarithm; Pointwise Mutual Information

NoSQL 51

See also database model; Extensible Markup Language; Resource Description Framework; triple; triple store

NPMI (Normalized Pointwise Mutual Information) 240-246

Object $\quad 45^{-46,6 o, 115}, 118 \mathrm{n} 28,120,133^{-134}$, 137, 218n79, 259, 307-308, 345-348, 354, 356

Object-oriented mapping 355

Objective variables $\quad 161,166-167,169$

OCHRE (Online Cultural and Historical

Research Environment) 314-315,

318-329, 333-334

OCR (Optical Character Recognition) 296-298, 304, 308-309, 322, 323n

oD (Open Data) 194, 218-219, 221, 344

oM (OntoMedia) 169n85, 336, 353-354, $357-361$
Online Cultural and Historical Research Environment (OCHRE) 314-315, 318-329, 333-334

See also data model; Extensible Markup Language; item; Java application client; Java Web Start; linked

Ontological class 349

Ontology $47,169 n 85,300 n 64-65,320$, 348-349, 353-355, 357-358, 361

See also Linked Data; upper ontology

OntoMedia (oM) 169n85, 336, 353-354, 357-361

See also cIDoc Conceptual Reference Model; ontology

Open Data (OD) 194, 218-219, 221, 344

See also Comma Separated Value; Linked Data; Linked Open Data

Open Richly Annotated Cuneiform Corpus

(Oracc) 198-200, 203-204, 225,

227-229, 232n32, 246, 253

See also annotation; ASCII Transliteration

Format; metadata; tag

See also in General Index Akkadian; Sumerian

Optical Character Recognition (OCR) 296-298, 304, 308-309, 322, 323n

See also abstract vector; algorithm; deep learning; features' vector; raster image; vector graphic

See also in General Index sign

Oracc (Open Richly Annotated Cuneiform Corpus) 198-200, 203-204, 225,

227-229, 232n32, 246, 253

Oracc-ATF 200

See also ASCII Transliteration Format;

Open Richly Annotated Cuneiform

Corpus

See also in General Index transliteration

Output vector 246-247

OWL (Web Ontology Language) 344, 345n21,

353,357

Oxford Text Archive $35^{2}$

Parser 227, 239, 293, 304

Parsing 154, 163, 182, 186, 293, 301, 329-330

Pennsylvania Sumerian Dictionary (ePSD)

$35^{2}$ 
Perseus Digital Library $\quad 219 n 82$

Pixel 296

Platform 48, 200, 238, 303, 317, 334

PMI (Pointwise Mutual Information) 225, 229, 232-233, 238-240, 242-244, 246, 249-252

Pointwise Mutual Information (PMI) 225, 229, 232-233, 238-240, 242-244, 246, 249-252

See also Artificial Neural Network; Word2vec

Potrace engine $\quad 304 n 83$

Pre-processing $\quad 166,174,184-185,197-198$

Predicate 46, 345-346, 348

Prosopography 210n6o, 329-332

Protocol 29-30, 340

Python 209, 213-214, 216, 218n79, 220, 238, 267-268, 293n41, 297n55, 306n88

Qualitative method (and related terms) 62, 162-163, 170, 186, 221, 226, 233

See also data mining; mixed method; quantitative analysis/method; $\mathrm{R}$; text mining

Qualitative data 94n29, 120-121

Quantitative analysis/method 94n29, 118, 162-163, 212, 216-217, 234, 257-258, 277

See also correspondence analysis; graph partitioning; machine-actionable data/ machine-readable data; mixed method; network analysis; qualitative method

Quantitative data $121,218,251$

Query/querying 46, 49, 196n $7,199,202,206$, 247, 285, 295, 315, 327-328, 331, 347

See also MySQL; Structured Query Language

R $\quad 154,162-163,167,182,184,186,318$ See also qualitative method

Ras Shamra Tablet Inventory (RSTI) 154n15, 314, 317, 318n16, 319-321, 325-329, 331, 333-334

See also Online Cultural and Historical

Research Environment; research database environment

See also in General Index Ras Šamra; tablet; transliteration

Raster image $\quad 296-297$
See also Optical Character Recognition

Recording 27-29, 33, 35-36, 38, 44, 46, 50, $55,297,302$

Recording system $\quad 26-27,29,33,36,38$, $44-45,48-49$

RDF (Resource Description Framework) 46-47, 51n98, 339-340, 344-348, 352, $360-361$

RDF Schema (RDFS) 344

RDF-XML 339

See also Extensible Markup Language; Resource Description Framework

RDFS (RDF Schema) 344

Regular expressions 205, 293

Relational data model 319

See also item

Relational database $\quad 47,202,204,345$

See also identifier; MysQL; querying; Structured Query Language; tuple

Research database environment 314

See also Ras Shamra Tablet Inventory

Resource Description Framework (RDF) 46-47, 51ng8, 339-340, 344-348, 352, $360-361$

See also data model; graph; HyperText Transfer Protocol Universal Resource Identifiers; relational database; Simple Knowledge Organization System; triple RSTI (Ras Shamra Tablet Inventory) 154n15, 314, 317, 318n16, 319-321, 325-329, 331, $333-334$

Scalable Vector Graphics (SVG) 300, 304, 307

See also EpiDoc; Extensible Markup Language

Scalar multiplication $\quad 284 \mathrm{n} 6$

See also vector

Scoring method 241

Scraper 204

See also machine-actionable data/ machine-readable data; Open Richly Annotated Cuneiform Corpus; tag; token

See also in General Index lemma Scraping 290n28

Self-Organizing Map (som) 65n2o-21, 94

See also Artificial Neural Network; multivariate analysis; node; unsupervised method 
Semantics $\quad 343,346 \mathrm{n} 25,361$

Semantic technologies $\quad 344$

See also algorithm; Artificial Intelligence; data mining; Natural Language Processing

Semantic unit 285, 286n14, 296, 298, 300-301, 304, 310

Semantic Web (sw) 46, 227, 336, 338-339, 343-344, 347

Semantic Web (sw) technologies 337, 339-341, 343-344, 348-349, 36o-361

See also Resource Description Framework; Simple Knowledge Organization System

Semantic website $171 n 92$

Semantics of a recording system $\quad 38$

See also arbitrary unit

Serialization format 299

Shading attribute 295

See also Manuel de Codage

See also in General Index sign

Simple Knowledge Organization System (skos) 46, 48ng1, 353

See also data cloud; Linked Data; machine-actionable data/machinereadable data; Resource Description Framework; Semantic Web

skos (Simple Knowledge Organization System) 46, 48ng1, 353

skosifying 48

See also Simple Knowledge Organization System

Social network analysis $\quad$ 195, 220, 227, 332

See also network graph

See also in General Index administrative texts

som (Self-Organizing Map) 65n20-21, 94 SPAD (Système Portable pour l'Analyse des Données) $\quad 97-98$

SPARQL $\quad 340,345,361$

Spatial semantic $\quad 69-70$

Special characters $\quad 285,286 \mathrm{n}_{13}, 287-288$

See also CAL Code

See also in General Index sign

SQL (Structured Query Language) 123n42, 196n7

Standardization/standardized 197, 200, 267, 299n59-6o, 306n86, 321
STAR project $\quad 47$

Statistical method $115,117-118,232,238,253$

STELLAR project 47

String 268, 347

Structured Query Language (SQL) 123n42, 196n7

See also MysQL; querying; relational database

Subject $\quad 46,345^{-}-348$

Subjective variables $161,169,178,181,185^{-186}$

Supervised method $\quad$ 260-261, 266

See also algorithm; unsupervised method SVG (Scalable Vector Graphics) 300, 304, 307 sw (Semantic Web) 46, 227, 336, 338-339, 343-344, 347

sw technologies (Semantic Web technologies) 337, 339-341, 343-344, 348-349, $360-361$

Syntactic elements 36

See also event

Système Portable pour l'Analyse des Données (SPAD) $\quad 97-98$

See also data mining; Graphical User Interface

Tag 42, 44, 163, 164n66, 165, 176, 184, 289n27, 302, 339

See also attribute; EpiDoc; Extensible Markup Language; markup; namespace; Text Encoding Initiative

TEI (Text Encoding Initiative)/TEI-XML 154, $162-167,185,203-204,289,302,326,35^{2}$

TEI-C (Text Encoding Initiative Consortium) $164,326 \mathrm{n}$

Term Frequency-Inverse Document Frequency (TF-IDF) $271 n_{34}$

Text data $\quad 165$

Text Encoding Initiative (TEI) 154, 162-167, 185, 203-204, 289, 302, 326, $35^{2}$

See also attribute; element; EpiDoc; interoperability

Text Encoding Initiative Consortium (TEI-C) $164,326 \mathrm{n}$

Text Mechanic Combination Generator Tool $207 \mathrm{n}_{52}$

Text mining $154,162-163,181-182,185^{-186}$, 196

See also qualitative method 
TF-IDF (Term Frequency-Inverse Document Frequency) 271n 34

Token 203-207

See also dictionary

See also in General Index lemma; sign

Tokenization/tokenization process 166, 197, 203, 204n 41

See also attribute; Text Encoding Initiative

See also in General Index sign; transliteration

Toolkit 238, 247-248, 283n2

Topological Weighted Centroid (TWC) 70-72, 74-75

Topological Weighted Centroid (TWC) mathematical approach 70 no

See also Topological Weighted Centroid

Tree-graph $66-67,69$

Triple 46, 49n95, 201-203, 211, 345-347, 36o

See also edge; network graph triples; node

See also in General Index tablet

Triple store $\quad 46,49 n 95,51 n 98$

See also triple

Tuple $47 \mathrm{n} 84$

TURTLE 340

TwC (Topological Weighted Centroid)

70-72, 74-75

TWC mathematical approach (Topological Weighted Centroid mathematical approach) $\quad 70{ }^{30}$

Unicode $285,287-288,300-301,308,310$, 328,351

See also mapping; Unicode Consortium; Unicode Transformation Format (UTF)-8

Unicode Consortium $\quad 328 n 34$

See also Unicode

Unicode Transformation Format (UTF)-8 289n25, 301068

See also EpiDoc

Universal Resource Identifiers (URIs) 302n72, 338-340, 344, 346, 348

See also HyperText Transfer Protocol; HyperText Transfer Protocol Universal Resource Identifiers; identifier; web

Unsupervised method 260-261, 266

See also algorithm; Self-Organizing Map; supervised method
Unweighted Pair Group Method with

Arithmetic Mean (UPGMA) 274-275

See also clustering method

UPGMA (Unweighted Pair Group Method with Arithmetic Mean) 274-275

Upper Ontology 320

See also ontology

URIs (Universal Resource Identifiers) 302n $72,338-340,344,346,348$

Variable(s) 61, 63-64, 66n26, 67n27, 69-72, 73n, 91n21, 99, 118, 120, 160, 162, 181, 185-186, 261n10, 337

Vector $\quad 65 \mathrm{n} 20,246-248,272,284 \mathrm{n} 6,297 \mathrm{n} 54$

See also Optical Character Recognition; Scalable Vector Graphics; scalar multiplication

Vector addition $\quad 284 n 6$

See also vector

Vector graph 310

Vector graphic 296, 300, 304

See also Optical Character Recognition; vector

Vector image $\quad 296$

Vector space $\quad 271,284 \mathrm{n} 6,285,305^{-306}$

See also scalar multiplication; vector; vector addition

Vectorization process 297

VerbNet 170

Vertex 73, 306

Visualization diagram $\quad 49 n 95$

W3C (World Wide Web Consortium) 46, $340,344-345,348,361$

Web 253, 318, 319n23, 338-339, 347

See also hyperlinks; HyperText Markup

Language; HyperText Transfer

Protocol; Linked Data; Universal

Resource Identifiers; World Wide Web

Consortium

Web of data 339,341

Web of documents 339

Web Ontology Language (OWL) 344, 345n21, 353,357

Weight $\quad 43,67 \mathrm{n} 28,73,201,206,208,211,240$, 247

See also edge; weighted 
Weighted 71, 73, 201-202, 211n61, 241, 243 See also Cytoscape; edge; Gephi; weight

Wizard 329-332

See also workflow wizard

See also in General Index lexicography

Word sense induction 226, 252

Word2vec 225, 229, 232-233, 242, 246-252 See also Artificial Neural Network;

Continuous Bag-of-Words model;

Natural Language Processing;

Pointwise Mutual Information; querying; vector

WordNet 164n65, 170

Workflow wizard 314, 329, 332 See also wizard

World Wide Web Consortium (W3C) 46, 340, 344-345, 348, 361

See also web
Writing system $\quad 321,327,328 n 34$

See also Online Cultural and Historical Research Environment

See also in General Index Akkadian;

logosyllabic; Sumerian

XDR (External Data Representation) 299

XML (Extensible Markup Language) 51ng8,

163-164, 203-204, 289n27, 299, 302n72,

303-305, 319, 334, 339-340, 347

XML annotation (Extensible Markup

Language annotation) 204

XML-Elamite standard $\quad 287$

See also human readable

See also in General Index Elamite; transliteration

XML-scheme $\quad 289$ 